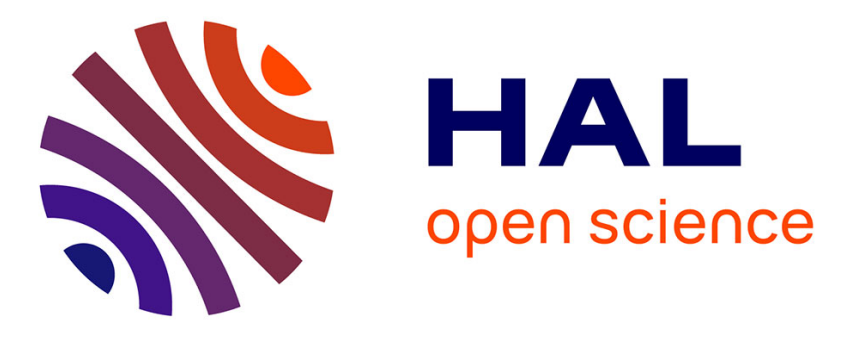

\title{
Outdoor augmented reality system for geological applications
}

\author{
Fakhr-Eddine Ababsa, Imane Zendjebil, Jean-Yves Didier, Joachim \\ Pouderoux, Jacques Vairon
}

\section{- To cite this version:}

Fakhr-Eddine Ababsa, Imane Zendjebil, Jean-Yves Didier, Joachim Pouderoux, Jacques Vairon. Outdoor augmented reality system for geological applications. IEEE/ASME International Conference on Advanced Intelligent Mechatronics (AIM 2012), Jul 2012, Kaohsiung, Taiwan. pp.416-421, 10.1109/AIM.2012.6265927 . hal-00744387

\section{HAL Id: hal-00744387 \\ https://hal.science/hal-00744387}

Submitted on 28 Jan 2019

HAL is a multi-disciplinary open access archive for the deposit and dissemination of scientific research documents, whether they are published or not. The documents may come from teaching and research institutions in France or abroad, or from public or private research centers.
L'archive ouverte pluridisciplinaire HAL, est destinée au dépôt et à la diffusion de documents scientifiques de niveau recherche, publiés ou non, émanant des établissements d'enseignement et de recherche français ou étrangers, des laboratoires publics ou privés. 


\title{
Outdoor Augmented Reality System For Geological Applications
}

\author{
F. Ababsa, Member, IEEE, I. Zendjebil, J.Y. Didier, J. Pouderoux, and J. Vairon
}

\begin{abstract}
Augmented reality has been shown to be useful in many application areas such as maintenance skills, urban planning, interior design and entertainment, etc.. The development of the mobile augmented reality in these last years is due to the evolution of the technology at various levels: from sensors (GPS, inertial sensor, etc.) to mobile devices (Tablet-PC, PDA, etc.). In this paper, we present a mobile augmented reality system dedicated to outdoor applications. It encompasses a localization process based on an assistance scheme and combining several data acquired from a hybrid sensor (camera, GPS and inertial sensor). The visualization and interaction are performed using a tablet-PC. This system has been tested in an application intended to geologists in order to monitor and supervise the restoration of a castle on a long term. This paper details various components of the system, some results that were obtained and how the whole application was evaluated through end-user.
\end{abstract}

\section{INTRODUCTION}

A ugmented Reality (AR) enhances our perception of the real world with virtual objects. The considered applications in this area are numerous, going from assistant tools for the experts (like in medicine, maintenance) to entertainment purposes for the general public. However, these applications are generally restricted to indoor and small-scale environments. Nevertheless, few research works were published in the 90's on outdoor augmented reality, such as the MARS project [1]. Since few years, we notice the increase of this kind of application. This is motivated by the progress made in terms of technology on handheld devices (Tablet PCs, PDAs and cell phones, etc.) and the development of new generation of localization sensors that are more accurate and smaller. Indeed, some of these sensors (like GPS receiver and accelerometers) are now integrated into the mobile devices like smartphones and allow achieving interesting outdoor AR applications. In addition, the advances made in the fields of visualization, interaction and localization have contributed in the development of the outdoor AR projects where the user has a small control on its working environment. The RAXENV (outdoor augmented reality applied to environmental science) project that we present here offers an assistance solution based on AR for

F. Ababsa is with the IBISC Laboratory, university of Evry, 40, rue du Pelvoux, 91020 Evry cedex, France; e-mail: ababsa@iup.univ-evry.fr.

I. Zendjebil, is with the IBISC Laboratory, university of Evry, 40, rue du Pelvoux, 91020 Evry cedex, France; e-mail: zendjebil@iup.univ-evry.fr.

J.Y. Didier is with the IBISC Laboratory, university of Evry, 40, rue du Pelvoux, 91020 Evry cedex, France; e-mail: didier@iup.univ-evry.fr.

J. Pouderoux is with the BRGM, 3 avenue Claude Guillemin, BP 36009, 45000 Orléans, France; e-mail j.pouderoux@brgm.fr

J. Vairon is with the BRGM, 3 avenue Claude Guillemin, BP 36009, 45000 Orléans, France; e-mail j.vairon@brgm.fr the geosciences. This project aims to develop a system combining complementary solutions of localization, visualization and interaction on a mobile device. The localization is used to estimate the point of view of the handheld camera and then to register the real and virtual worlds. The user can interact in real time with the rendered scene which is displayed on a wearable tablet-PC.

In this paper, we describe the system proposed in the RAXENV project. We present each component including: localization, visualization and interaction. The paper is organized as follows. In the next section, we briefly present existing outdoor augmented reality systems. Section 3 overviews the RAXENV project and its objectives. In section 4 describe the hardware and software architecture of the designed system. The details functioning of the whole system are provided in section 5. Experiments conducted in real conditions and obtained results are presented in section 6. Finally, we conclude in section 7 and suggest some future works.

\section{RELATED WORKS}

During the last years, outdoor AR projects have proliferated over the world. These works focus on different areas such as military, civil engineering or cultural heritage. The existing applications in this field can be generally subdivided into two categories:

- Navigation applications which provide to the user relevant information in order to help him to evolve in his environment;

- Assistance applications which assist the users in his task (for example inspection task, etc.).

The first developed applications in this area had for main objective to demonstrate the feasibility of an AR system in outdoor environments. For example MARS [1] and Tinmith [2] projects have proposed original hardware and/or software architectures in adequacy with outdoor AR needs. The MARS project prototyping equipment has inspired several other projects such BARS project [3] which was developed for the military navigation. In the Tinmith project, the researchers have proposed hardware/software architectures managed by the Unix operating system. All theses systems were equipped by large backpacks holding the processing unit (laptops) which is connected to various types of sensors, such as GPS receiver and inertial sensor. Also, the processing unit can in some case communicate with remote database servers using $\mathrm{WiFi}$ as in the ARCHEOGUIDE project [4]. The display devices were either see-through display or terminals. However, in recent years, mobile handheld device provides a significant gain in computational 
power, autonomy and miniaturization. It allows replacing the processing unit and displaying device by a single processing unit, which is also used for visualization. They are more and more used for outdoor AR applications. This is the case of the Vidente [5] and IpCity projects [6] where a UMPC (Ultra-Mobile PC) is used. Other applications have used PDAs or mobile phones as it is the case in the TimeWarp project (Herbst, 2008). Actually, with the emergence of new generation of mobile devices integrating the navigation sensors (accelerometer, GPS, etc.), new AR applications are proposed for mainstream user to help him in his common life, like for example to guide him to the closest metro stations or to find an interesting restaurant. The progress made in this filed allows the development of new AR systems where the visual augmentations are not only restricted to simple annotations or avatars. Thus, the recent mobile outdoor AR systems provide the access to remote databases such as Geographic Information Systems (GIS) and represent knowledge in visual form. For example in RAXENV system, the geologist can view, on site, the geological sections superimposed on the volcano view. In addition, RAXENV uses new interaction methods which facilitate the data manipulation by the end-users and thereby enriches the data bases (GIS, CAD models, etc.) in real time. Various challenging issues were addressed in the RAXENV project; they are detailed in the next sections.

\section{RAXENV PROJECT}

RAXENV is an exploratory project whose main objective is to demonstrate the feasibility of AR paradigm applied to the environmental science and technology such as geology. In this context, several challenges have been identified in order to realize an efficient system able to:

- Localize itself using heterogeneous sensors (such as GPS, inertial and camera) and a priori knowledge on the environment (geo-referenced 3D models, Geographic Information Systems (GIS), digital terrain models (DTM)).

- Offer features to the user in order to visualize and query the local or remote geological data, using appropriate interaction methods with mobile devices.

The RAXENV system was designed for different scenarios involving different needs and skills in the field of environment sciences:

- An instrumented scenario (the castle of Saumur) to manage the monitoring of rebuilding a rampart.

- An urban scenario to manage sewerage network based on geo referencing data provided by a GIS.

- A panoramic scenario for geology applications to see geological survey map, or communication sites.

\section{SYSTEM OVERVIEW}

\section{A. Hardware platform}

The RAXENV main hardware component is a tablet-PC (CORE 2 DUO U7700) that is simultaneously the processing unit and the display terminal. The Tablet-PC is connected to a localization system relying on a hybrid sensor (figure 1). The hybrid system contains a GPS receiver, A Trimble GPS Pathfinder ProXT receiver, worn by the user. The ProXT receiver integrates a multipath rejection technology providing sub meter accuracy. Its rate update is about $1 \mathrm{~Hz}$. The ProXT receiver uses a Bluetooth wireless connection to communicate with the computer. Add to the GPS receiver, the hybrid sensor is composed of a camera coupled with an inertial sensor which is rigidly attached. The inertial sensor, a Xsens MTi, contains gyroscopes, accelerometers and magnetometers. The advantage of the MTi is that it incorporates an internal digital signal processor which runs a real-time sensor fusion algorithm providing a reliable 3D orientation estimate. Data from MTi are synchronously measured at $100 \mathrm{~Hz}$. For the vision, we opted for a USB uEye UI-2220RE industrial camera with $8 \mathrm{~mm}$ focal length which is extremely compact, low-cost and well adapted for outdoor environments. Colour images with resolution of $768 \times 576$ pixels at a frame rate of $25 \mathrm{~Hz}$ are streamed to the tablet-PC using a USB 2.0 connection.

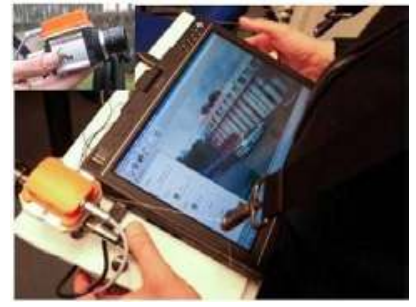

(a)

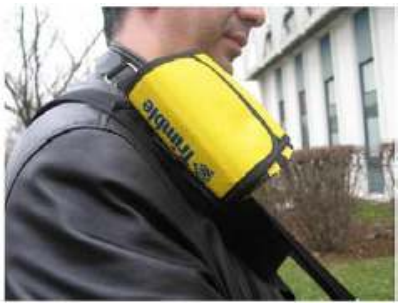

(b)
Fig. 1. Hardware platform of Raxenv Project. (a) Tablet PC, camera and inertial sensor (b) GPS receiver.

For interaction, 4 Phidgets [7] components are connected to the platform. Phidgets are a set of small analogical building blocks which can be plugged on a PC through USB and easily monitored and controlled using an easy to use API. We found that Phidgets give a very efficient way to enrich the plateform prototype by offering the user (in mobility with his only fingers to interact) haptic devices to accomplish some interaction tasks with the system.

\section{B. Software platform}

There are three different software parts integrated inside RAXENV system. A database representing the 3D model of the real environment is used to extract a priori knowledge. The sensors and the database are connected to libraries that are developed with our ARCS framework (Augmented Reality System Component) which is using a componentbased approach. So, components are modeled as objects with specific inputs and outputs called respectively slots and signals. An AR application is modeled as a set of nominal states (e.g. initialization, sensor calibration, tracking, reinitialization, etc) that share the same components. Depending on the current state of the application, components may be configured differently and connected to each other in another scheme. Thus, it allows the data-path between components to be configured differently, each one of these configuration (also called sheets) corresponding to a state. In order to control the application we used a finite state 
machine which also can communicate with the sheet components. In technical terms, components are written in $\mathrm{C}++$ and stored inside dynamic libraries loadable at runtime by the ARCS engine. In order to simplify application descriptions, composite components, represented in an XML format, are allowed to be composed using native components or composite components. The ARCS engine has several functionalities: it parses XML descriptions of $\mathrm{AR}$ applications, loads dynamic libraries, instantiates components and finally controls the application behaviour through a finite state machine. The ARCS framework also proposes a graphical editor in order to visually program and configure an application. The whole framework heavily relies on Qt3, which has XML capabilities and possesses an implementation of the signal/slot mechanism. ARCS contain about 120 components implementing data acquisition, image processing, graphical engine as well as some logical glue capabilities. In addition, the database is used by another framework called Elkano developed by Iparla research group. Elkano is a 3D engine of an object-oriented scene graph compatible with OpenGL and OpenGL ES and allows to quickly setting up visualization and interaction techniques for heterogeneous devices (PC, mobile phones, etc.) Written in $\mathrm{C}++$, Elkano can be easily extended through a plugging mechanism and easily integrated in any application to manage 3D contents. Indeed, the pluggin mechanism is intended to provide access to many kinds of "external" processing through a consistent / generic interface.

The GUI of the RAXENV application was designed in $\mathrm{C}++/ \mathrm{CLI}$ using the WinForms.NET API (Figure 2). There is only one main application for all the scenarios but small changes on the GUI are done according the specificity of each scenario needs.

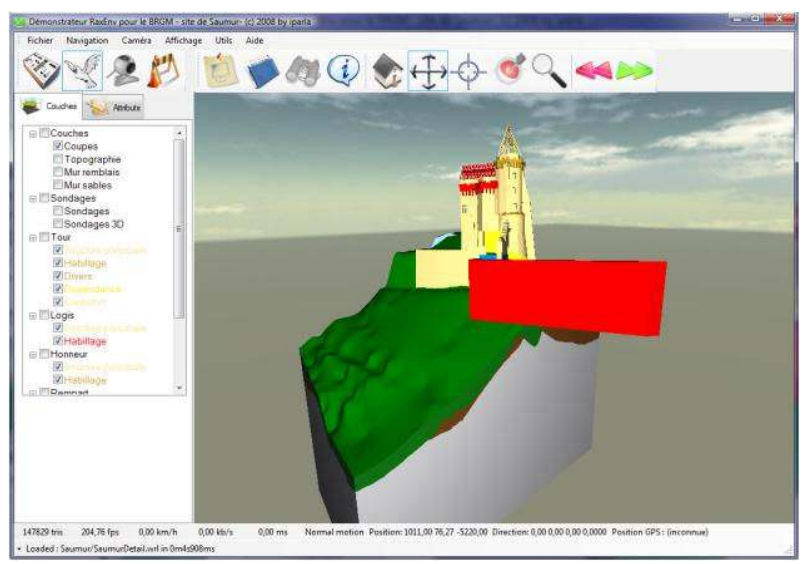

Fig. 2. Raxenv User Interface

\section{FUNCTIONAL REQUIREMENTS}

Figure 3 shows the data flow in the RAXENV platform. The project has focused on different aspects namely: real/virtual registration, access to remote data in a mobility context, visualization and interaction. The developed techniques include principally:

- A localization system based on multi-sensors technology
- A visualization method for representing subsurface objects (surveys, pipes, cavities, etc.) in AR context;

- An interaction approach suitable for mobile and handheld AR system.

The developed libraries concern the localization process and the sensors interfacing. The localization library contains the pose estimation approaches using data from the sensors and the database. Other libraries interface the sensors and forward the acquired data to the localization library. The estimated camera poses and the sensors data are then provided to the Elkano platform which performs the rendering of the augmented scene. This is visualized on the screen of the Tablet PC. Moreover, several phidgets are connected the Tablet-PC and allow the interaction with the visualized 3D data.

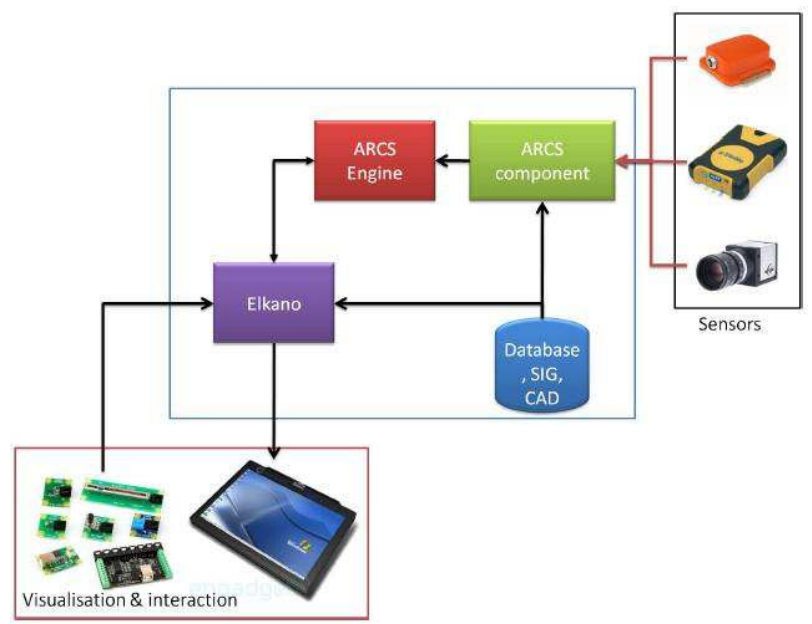

Fig. 3. Data flow in the RAXENV system

\section{A. 3D Localization}

The localization process is crucial for AR applications. Most AR systems, mainly those based on video see-through, use vision-based approaches to estimate the camera pose [8][9][10]. However, in outdoor environments, these approaches are sensitive to work conditions such as brightness changes, occlusions and sudden motions. For this reason, these applications converge to use hybrid sensor systems to overcome the drawbacks of using a single type of sensor (i.e. camera) and increase the robustness and the accuracy. The localization module used in the RAXENV platform is combined with data provided by the camera, the GPS receiver and the inertial sensor. it operates using an assistance scheme. The used approach is described in detail in [11]. Briefly, the assistance scheme subdivides the localization system into two parts: a main subsystem and an assistance subsystem. The main subsystem is vision-based approach for markerless tracking. 3D salient points extracted from the 3D model are matched with the first frame using a semi-automatic approach based on the SURF descriptor [12]. Such matched points are then tracked through the video stream using the KLT algorithm [13]. When the vision-based tracking fails because of occlusion or blur in images, the system switch automatically to the assistance subsystem, called Aid-Localization (AL) [14], including the GPS 
receiver and inertial measurement. In this case the AL replaces the camera to provide an estimation of the localization until the vision process is able to regain control. For that, the data from GPS and inertial sensor are transformed in the world reference frame defined during the calibration phase [15]. Since our system follows an assistance scheme, we will decompose it into four states:

- Init state: the system initializes itself using a semiautomatic approach;

- Vision predominance state: the system uses a visionbased approach to localize itself.

- AL predominance state: in this state, the system uses AL subsystem to estimate the localization;

- Reinit state: through this state, the system tries to reinitialize the vision after its failure.

The system switches from one state to another according to different criteria using the finite state machine (see figure 4) formalism which is mainly used in the theory of computability and formal languages.

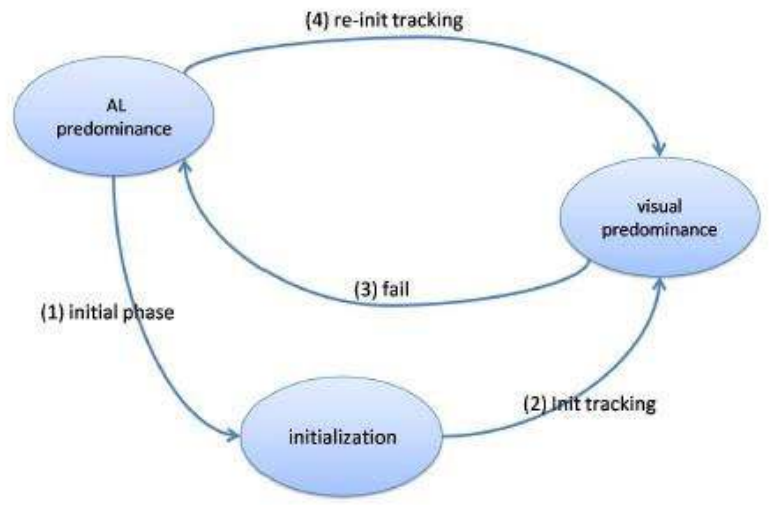

Fig. 4. The proposed state machine for 3D localization

\section{B. Visualisation and $3 D$ Interaction}

RAXENV project involves a huge quantity of $2 \mathrm{D}$ and $3 \mathrm{D}$ georeferenced data which must be graphically represented on the screen. We achieved this thanks to the Elkano framework. The different data sources are managed by layers which can be shown or hidden like in a classical GIS. We mainly distinguished 3 main data sources:

1. 3D models and scenes which can be used to represent purely visual elements manually modelled from real world objects (buildings, walls, vegetation, road, etc.);

2. GIS data which can describe, using $2 \mathrm{D}$ vectors and some metadata, ground and underground elements. The 3D representation of such objects is achieved in a procedural way by extensions of Elkano;

3. The geological data, one of the most important components of the project. One of these data is the geological model which is represented by a simplified form called "multi-layer" (a stack of 2.5D grids where each grid represents the elevation of the highest of lowest limit of a given geological layer).

To represent those layers, we developed an algorithm which virtually represents a volumetric model from the set of grids. The considered resolution of the grids is about $1 \mathrm{~m}$. Indeed, borders of the geological model are reconstructed on the fly by generating a vertical mesh connecting the layers two by two. This technique also allows us to offer different configurations of cutting planes (like excavation or drill holes) which are very useful tools to analyze geological models for geologists as illustrated by figure 5 .
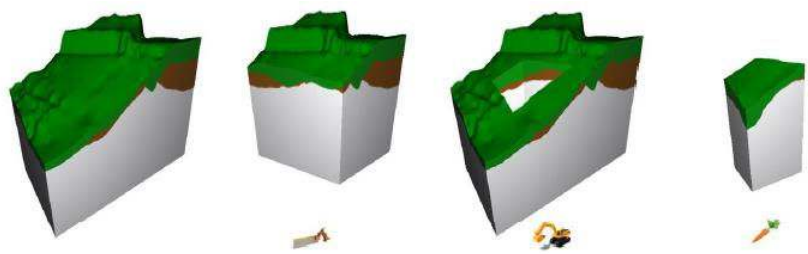

Fig. 5. Different cutting planes configuration on the geological model.

In the context of an augmented reality application, the visualization of the captured video frame in real time is of course one of the main need. We offer the user to show this image in foreground or in background of the 3D model with an adjustable transparency level. During the calibration step, the wireframe model and the points are shown similarly. We should also mention that 3D objects are mutable: the user can select each of them and edit their rendering properties (colour, transparency, etc.) or, for GIS objects, their metadata which can also have a direct effect on their graphical representation (for instance the radius or material of a pipe). The augmented view (mixing the camera image and the 3D view of the georeferenced objects) can also be deactivated to offer the user a virtual navigation in the $3 \mathrm{D}$ scene around him. The user can thus step back and put into situation elements of interest. To navigate the virtual scene, the prototype implements different interaction techniques specifically designed for a multitouch screen. Among them we can cite Navidget [16], ScrutiCam [17], a continuous go to technique and a binoculars-like mode which allows to quickly observe a particular point from the current point of view. As mentioned earlier, some interaction tasks can be done using one of the 4 Phidgets components chosen for the prototype. For instance, a touch button can be used to switch between the augmented view and the free virtual navigation, a slider can be used to go forward/backward in virtual navigation or to move a cut plane in a given direction, a button allows changing the transparency of a selected 3D element in the scene, etc.

\section{RESULTS}

Numerous experiments are done in order to evaluate the performances of the RAXENV platform, in particular the software frameworks assessment, the localization accuracy and the end-user reviews.

\section{A. Software framework assessment}

RAXENV enabled to assess the ARCS framework in the context of mobile augmented reality. Amongst the interesting points that we have observed, we can cite:

- the use of a component based architecture makes the composition of applications quite easy; 
- decomposing the application in several sheets and using a state machine in order to control the whole system is very powerful to manage hybrid sensor tracking.

\section{B. 3D Localization results}

The localization approach used in RAXENV project was tested in different situations. The results of these experiments are described in details in [11]. We will focus mainly on the results obtained on tests conducted in the Saumur castle using the whole system. The tests were performed in the courtyard outside the castle, where we have a priori knowledge of the partial 3D model. The hybrid sensor was calibrated in advance using a set of data (GPS positions, orientations of the inertial sensor and camera's poses) collected on the site. In the figure 6 , we can observe the registration of the $3 \mathrm{D}$ model represented the castle on the actual view. This registration is obtained from the poses estimated by the localization module and returned to the visualisation module which performs a rendering of the augmented scene.

We obtain a mean reprojection error around 1.67 pixels with a standard deviation about 2.37. The mean error of the orientation obtained from inertial sensor is about $(\mathrm{x}=0 ; 27$; $\mathrm{y}=0: 41 ; \mathrm{z}=0: 24)$ with standard deviations of $(0: 28 ; 0: 43 ; 0: 25)$. In generally the system behaviour is correct and obtained results are satisfactory especially when the position errors are corrected using the error prediction module. Concerning the position obtained from the GPS receiver, the mean error is around $(1: 84 \mathrm{~m} ; 1: 48 \mathrm{~m})$ with standard deviation equal to $(1: 11 \mathrm{~m} ; 0: 96 \mathrm{~m})$ respectively on $\mathrm{x}-$ axis and y-axis. The GPS receiver is used only for 2D positioning. In fact, the elevation returned by the GPS is less accurate. To supplement the GPS data, the position on the $\mathrm{z}$ axis is set at the average size of the user with the elevation of the ground, this correspond to the position of the sensors handhold by the user.
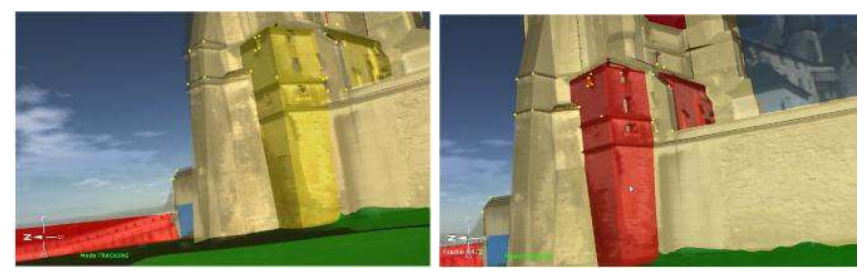

Fig. 6. Example of registration on the castle site

\section{AR Contributions: end-users reviews}

The RAXENV prototype has been tested on the site of the castle of Saumur. No in-depth user study has been done yet but the first reviews and experiments on the site were very encouraging. The end-users (Geologists and Geotechnical experts) have tested the Raxenv tool during the design process and also in real working conditions. They feel very interesting to interact with the 3D geological model when it is mixed with the camera video. They found the AR view very useful to have a better understanding of the underground structures. The use of Phidgets was reported to be a very good idea because it avoids the imprecise contact with the touch screen.

\section{CONCLUSION}

In this paper, we have presented the RAXENV platform, an augmented reality system which aims to provide an assistance tool for geoscientists to help them in their outdoor tasks. A great care has been take in the design of the final application to offer the mobile user the possibility to interact with the model using haptic or touch screen interfaces. The project demonstrated the feasibility and highlights the interest of augmented reality in the context of trades applications or general public from geosciences. The demonstrators and prototypes offer to the end-user innovative ways to navigate, interact and interview data implemented. However, the work is underway to be completed mainly concerning the 3D localization. Indeed, the used vision-based approach is dependent on the accuracy of the 3D model. The suggested idea is to move towards SLAM approaches to break away from the use of the 3D model mostly in urban environments where we have only the data extracted from GIS and sensors.

\section{ACKNOWLEDGEMENTS}

This work is supported by the RAXENV project funded by the French National Research Agency "ANR".

\section{REFERENCES}

[1] T. Hollerer, S. Feiner, T. Terauchi, G. Rashid, and D. Hallaway, "Exploring mars: Developing indoor and outdoor user interfaces to a mobile augmented reality system". Computers and Graphics, vol. 23, $\mathrm{n}^{\circ} 6, \mathrm{pp} .779-785.1999$

[2] W. Piekarski., and B. Thomas, "Tinmith-evo5- architecture for supporting mobile augmented reality environments". in Proc of IEEE Int. Symp. on Augmented Reality, pp. 177-178. 2001

[3] M.A. Livingston, D. Brown, S.J. Julier, and G.S. Schmidt, "Military applications of augmented reality". NATO Human Factors and Medicine PanelWorkshop on Virtual Media for Military Applications. 2006.

[4] T. Gleue, and P. Dahne, „Design and implementation of a mobile device for outdoor augmented reality in the archeoguide project". In Conference on Virtual reality, archeology, and cultural heritage, pp. 161- 168. ACM Press. 2001

[5] G. Schall, E. Mendez, E Kruijff, E Veas, V. Junghanns, B. Reitinger, and D. Schmalstieg, Handheld augmented reality for underground infrastructure visualization. Personal and Ubiquitous Computing. 2008 .

[6] S. Markus, and S. Dieter, Urban sketcher: Mixed reality on site for urban planning and architecture. In 6th International Symposium on Mixed and Augmented Reality, pp. 27-30. 2007

[7] S. Greenberg, and C. Fitchett, "Phidgets: easy development of physical interfaces through physical widgets". In Symp. on User interface software and technology, pp. 209-218. ACM Press. 2001

[8] F. Ababsa, M. Mallem "Robust Camera Pose Estimation Combining 2D/3D Points and Lines Tracking". In the Proc. of the 2008 IEEE International Symposium on Industrial Electronics (ISIE'08). Cambridge, UK, pp. 774-779, 2008.

[9] F. Ababsa, M. Mallem. "A Robust Circular Fiducial Detection Technique and Real-Time 3D Camera Tracking”. International Journal of Multimedia (JMM). Academy Publisher, vol. 3, no. 4, pp. 34-41. 2008.

[10] J-Y Didier, F. Ababsa, M. Mallem. "Hybrid Camera Pose Estimation Combining Square Fiducials Localisation Technique and Orthogonal 
Iteration Algorithm". International Journal of Image and Graphics (IJIG). Vol. 8, no. 1, pp. 169-188. 2008

[11] I. Zendjebil, F. Ababsa, J.Y Didier, and M. Mallem, "Large scale localization for mobile outdoor augmented reality applications". International Conference On Computer Vision Theory and Applications. Springer, editor. 2011

[12] H. Bay, A. Ess, T. Tuytelaars, and L.V. Goo, "Surf: Speeded up robust features". Computer Vision and Image Understanding (CVIU), 110(3), pp. 346-359. 2008

[13] B. Lucas, and T. Kanade, "An iterative image registration technique with an application to stereo vision". In Proceedings of the 7th International Joint Conference on Artificial Intelligence, pp. 674-679. 1981

[14] I. Zendjebil, F. Ababsa, J.Y. Didier, and M. Mallem, "On the hybrid aid-localization for outdoor augmented reality applications. In Symposium on Virtual reality software and technology, pp. 249-250. ACM Press. 2008

[15] I. Zendjebil, F. Ababsa, J.Y. Didier, and M. Mallem, "A gpsimucamera modelization and calibration for $3 \mathrm{~d}$ localization dedicated to outdoor mobile applications". International Conference On Control, Automation and system. 2010

[16] I. Herbst, A.K. Braun, R. McCall, and W. Broll, "Timewarp: Interactive time travel with a mobile mixed reality game". In International Conference on Human Computer interaction with Mobile Devices and Services, Amsterdam, The Netherlands. ACM Presss. 2008

[17] M. Hachet, F. Decle, S. Knodel, and P. Guitton, "Navidget for easy 3d camera positioning from $2 \mathrm{~d}$ inputs". In Proceedings of IEEE 3DUI Symposium on 3D User Interfaces, pp 83-88. 2008 